\title{
Expression of a ribosome-inactivating protein gene in bitter melon is induced by Sphaerotheca fuliginea and abiotic stimuli
}

\author{
Jun Xu $\cdot$ Hualin Wang $\cdot$ Jianming Fan
}

Published online: 28 December 2007

(C) Springer Science+Business Media B.V. 2007

\section{Erratum to: Biotechnol Lett (2007) 29:1605-1610 DOI 10.1007/s10529-007-9433-3}

In the original paper, we claimed to have cultivated Sphaerotheca fuliginea on laboratory medium. This was in error as this fungus cannot be grown on such media. The organism concerned for this aspect of the work was Fusarium solani. In all other aspects of the work, the fungus as named was used.

Accordingly, in the Abstract, the third sentence should read:

"The ribosome-inactivating protein was expressed in $E$. coli and, when purified, it inhibited the growth of Fusarium solani."

In the Methods and materials section (p. 1607), 'Antifungal assay', this should now read:

The online version of the original article can be found under doi:10.1007/s10529-007-9433-3.

J. Xu $\cdot$ H. Wang

State Key Laboratory of Virology, Wuhan Institute of Virology, Chinese Academy of Sciences, Wuhan 430071, China

J. Xu

Graduate School of the Chinese Academy of Sciences, Beijing 100039, China

J. Fan $(\bowtie)$

The Laboratory of Toxicology, College of Public Health, Zheng Zhou University, Zheng Zhou 450052, China e-mail: 5746067@sohu.com
"Fusarium solani was inoculated on the center of "

In the Results section (p. 1607, left hand column, line 37), this should now read:

"The antifungal activity of the purified recombinant RIP was tested by determining its inhibitory effect on the hyphal extension of Fusarium solani (Fig. 2)."

Accordingly, the legend to Figure 2 should now read:

"Inhibition assay of hyphal extension of Fusarium solani growing on potato dextrose agar medium by purified recombinant RIP. Fusarium solani was inoculated on the center of a potato/dextrose/agar plate."

In the Discussion, the third sentence (p. 1609, left hand column, lines 19-22) should read:

"The purified recombinant RIP was found inhibiting the growth of Fusarium solani in vitro. In the present investigation, a RIP gene was cloned from plant leaves and expressed in $E$. coli and its antifungal activity against Fusarium solani pathogen was determined."

We apologise for giving potentially misleading information which was caused by a simple laboratory error in labelling respective fungal cultures. 